\title{
Living with dementia: increased level of caregiver stress in times of COVID-19
}

\author{
Gabriela Cohen, María Julieta Russo, Jorge A. Campos, and Ricardo F. Allegri
}

Fundación para la Lucha contra las Enfermedades Neurológicas de la Infancia, Memory and Ageing Center, Department of Cognitive Neurology, Neuropsychology and Neuropsychiatry (FLENI), Buenos Aires, Argentina

ABSTRACT

COVID-19 pandemic in Argentina has affected the care of older adults with dementia deeply. Our objective was to study how the obligatory social isolation affected stress caregiver and burden of care of family members of subjects living with dementia in the community after the initial 4 weeks of quarantine in our setting. We did a questionnaire survey among 80 family caregivers of persons with Alzheimer's disease (AD) or related dementia collected on April 2020. We designed a visual analog scale to test the level of the burden of care. Characteristics of people with dementia and their caregivers were analyzed with descriptive (mean, standard deviation, frequency and percent) and inferential statistics (chi-square test). The sample included older adults (mean age: $80.51 \pm 7.65$ ) with different stages of dementia. Family was the primary provider of care in $65 \%$. Overall, COVID-19 confinement increased stress caregiver independently of the dementia stage, but those caring for severe cases had more stress compared to milder forms of the disease. Other findings were that half of the subjects with dementia experienced increased anxiety and that most family members discontinued all sort of cognitive and physical therapies. Family members' main concerns were for severe dementia cases, fear of absence of the paid caregiver during the epidemic, and for mild cases fear of spreading the disease while assisting patients with instrumental activities. A partnership between departments of public health, care workers and families must be planned to guarantee continuity of care during these unique COVID-19 times.

Key words: Alzheimer's disease (AD), dementia, outpatient

\section{Introduction}

Coronavirus disease (COVID-19) pandemic has deeply affected the care that older adults with Alzheimer's disease $(\mathrm{AD})$ and related disorders received in Argentina. Even though circulation for family caregivers of subjects with dementia was one of the few exceptions allowed by the government (Ministerio de la Nación, 2020), we observed that most family members decided to stop visiting their relatives from fear of spreading the disease.

COVID-19 epidemic is causing a radical change in the model of dementia care. Before this pandemic, engaging in social activities, performing cognitive and physical activities, and having a productive daily routine have been the mainstay therapy (Austrom et al., 2018). To relief caregiver stress, literature has shown that multicomponent

Correspondence should be addressed to: Gabriela Cohen, MD, Cognitive Department, Capital Federal, Montañeses 2325, 8th Floor, Buenos Aires 1428, Argentina. Phone: + $5491148023788 /+5491157281522$. Email: gcohen@fleni.org.ar, dra.gcohen@gmail.com. Received 28 Apr 2020; revision requested 25 Jun 2020; revised version received 10 Jul 2020; accepted 19 Jul 2020. First published online 30 July 2020. strategies such as avoiding isolation, attending family and group support meetings, and sharing the burden of care with other family members were useful (Hughes et al., 2014). Now, in times of COVID-19, we recommend the most strict social isolation, especially for older patients with dementia and other comorbidities who have the highest risk for severe COVID-19 disease and mortality (Emami et al., 2020).

Previous quarantines in human history had a negative psychological impact on outcomes such as anger, depression, and loneliness in the general population (Brooks et al., 2020), but the effects on the well-being and standard care of subjects with dementia living in the community are not well studied. The objective of our research was to study to what extend mandatory social isolation affected the stress and burden of care of family members caring for subjects with dementia after the initial 4 weeks of quarantine and to study the relationship between the severity of the dementia, measured with the Clinical Dementia Rating (CDR) (Hughes et al., 1982), and the impact of the negative effects of quarantine in our setting. 


\section{Material and methods}

This brief report is based on data of a questionnaire survey among 80 family caregivers of persons with $\mathrm{AD}$ or related dementia, collected during 2 days of April 2020 from the Aging and Memory Center at FLENI in Buenos Aires. All participants had a comprehensive diagnostic evaluation before the isolation period by a member of the team with experience in Cognitive Neurology.

We specifically designed for our study an online questionnaire with an accessible format to be completed by family members of persons with $\mathrm{AD}$ or related dementia. The questionnaire had simple and easy to answer questions. It was not intended to replace medical consultations, validate tests, or make accurate neuropsychiatric diagnoses. Items asked included demographic characteristics of both subjects with dementia and family members and problems of management, rehabilitation, and care that subjects experienced during the first 4 weeks of the coronavirus quarantine in our setting.

To test the presence of anxiety, we asked caregivers two simple questions: "Did your relative with dementia experienced anxiety before the pandemic?" and "Do your relative with dementia experienced anxiety during the pandemic?

To measure the amount of burnout and stress that a family caregiver experienced, we asked the following question: "How much stress related to the care of your family member with $\mathrm{AD}$ are you experiencing during this quarantine?" For this purpose, we designed a visual analog scale, with a continuous range from none to an extreme amount of stress. Based on the obtained score, the results were transformed into three categories: low, medium, and high level of burden.

To overcome some of the limitations imposed by conventional pretest-posttest self-report measures, a retrospective pretest-posttest design was utilized. We made this selection since a retrospective pretestposttest design is a convenient and valid method for measuring self-reported change. This method has shown to reduce response shift bias with more accurate assessments of actual effect and provides comparison data in the absence of "pre" data (Nimon, 2014).

\section{Analyses}

The characteristics of people with $\mathrm{AD}$ or related dementia and their caregivers were analyzed with descriptive (mean, standard deviation, frequency, and percent) and inferential statistics. To evaluate the association of dementia stage, caregiver burnout, and main clinical data, chi-square tests (dependent variable: dementia stages based on CDR score) were used. The main results were presented as percentages of studied variables to provide a straightforward summary statistic easily understood. Statistical analyses were performed using SPSS 21.0, with statistical significance set at $p<.05$.

\section{Ethics}

A letter with an invitation to participate in the survey was mailed to family members explaining the objectives of the research study. Confidentiality of family members and subjects with $\mathrm{AD}$ were strictly preserved through all phases of the survey process and forever after. According to the Argentina law, no approval of a Medical Ethics Committee is needed for a survey research that does not involve any intervention.

\section{Results}

Characteristics of participating caregivers and persons with $\mathrm{AD}$ or related dementia are reported in Table 1 . Twenty-three family caregivers $(28.8 \%$ of the total sample) had a relative in the initial stage of dementia $(\mathrm{CDR}=1), 33(41.3 \%)$ in an intermediate stage $(\mathrm{CDR}=2)$, and $24(30.0 \%)$ had a relative who have severe dementia $(\mathrm{CDR}=3)$. The most frequent subtype of dementia was $\mathrm{AD}$, followed by mixed $\mathrm{AD}$, with $61.3 \%$ and $20.0 \%$, respectively. The average age of the sample was $80.51 \pm 7.65$, and the average education was $13.95 \pm 4.94$. Women accounted for $62.5 \%$ of persons with dementia.

Of our sample, $43.8 \%$ of people with $\mathrm{AD}$ or related dementia experienced anxiety before the coronavirus pandemic without a statistical difference along the spectrum of severity of the disease based on CDR score $\left(\chi^{2}(2)=.281, p=.869\right)$. Fortyeight percent of the sample reported an increased level of anxiety in the context of containment for COVID-19. Of these 39 patients, 12 needed an increase in antipsychotics doses and 7 needed an increase in benzodiazepines doses.

Before the pandemic, $68.9 \%$ of the patients underwent at least a weekly session of ambulatory neurological rehabilitation therapy (average: 1.25 sessions per patient). These included physical therapy $(61.3 \%)$, occupational therapy $(23.8 \%)$, and cognitive rehabilitation $(40.1 \%)$. We analyzed if patients with intermediate-severe dementia received more physical therapy rehabilitation than patients with mild dementia and we found no statistically significant difference $\left(\chi^{2}(4)=8.314, p=.08\right)$. More than $90 \%$ of the patients discontinued therapies during the pandemic. Also, this percentage did not differ significantly for persons in the three stages of dementia $(p>.05)$ 
Table 1. Characteristics of participating caregivers and persons with Alzheimer's disease or related dementia (in percentages)

\begin{tabular}{|c|c|c|c|c|c|}
\hline \multirow[b]{2}{*}{ Variables } & \multicolumn{3}{|c|}{ CDR } & \multirow[b]{2}{*}{$\chi^{2}$} & \multirow[b]{2}{*}{$p$} \\
\hline & 1 & 2 & 3 & & \\
\hline \multicolumn{6}{|l|}{ Part I: Persons with Dementia } \\
\hline \multicolumn{6}{|l|}{ Age } \\
\hline$<45$ years old & $33.30 \%$ & $11.10 \%$ & $55.60 \%$ & 2.996 & .558 \\
\hline $45-65$ years old & $23.80 \%$ & $57.10 \%$ & $19.00 \%$ & & \\
\hline $65-85$ years old & $25.00 \%$ & $37.50 \%$ & $37.50 \%$ & & \\
\hline$\geq 85$ years old & $0.00 \%$ & $100.00 \%$ & $0.00 \%$ & & \\
\hline \multicolumn{6}{|l|}{ Gender } \\
\hline Male & $23.30 \%$ & $43.30 \%$ & $33.40 \%$ & 0.718 & .698 \\
\hline Female & $32.00 \%$ & $40.00 \%$ & $28.00 \%$ & & \\
\hline \multicolumn{6}{|l|}{ Diagnosis } \\
\hline $\mathrm{AD}$ & $28.60 \%$ & $44.90 \%$ & $26.50 \%$ & 10.455 & .107 \\
\hline Mixed AD & $37.50 \%$ & $43.80 \%$ & $18.80 \%$ & & \\
\hline Vascular dementia & $42.90 \%$ & $28.60 \%$ & $28.60 \%$ & & \\
\hline Others & $0.00 \%$ & $25.00 \%$ & $75.00 \%$ & & \\
\hline \multicolumn{6}{|l|}{ Caregiver } \\
\hline Paid caregiver & $21.40 \%$ & $35.70 \%$ & $42.90 \%$ & 3.497 & .174 \\
\hline Family caregiver & $32.70 \%$ & $44.20 \%$ & $23.10 \%$ & & \\
\hline \multicolumn{6}{|l|}{ Part II: Family Caregivers } \\
\hline \multicolumn{6}{|l|}{ Age } \\
\hline$<45$ years old & $33.30 \%$ & $11.10 \%$ & $55.60 \%$ & 7.514 & .276 \\
\hline $45-65$ years old & $23.80 \%$ & $57.10 \%$ & $19.00 \%$ & & \\
\hline $65-85$ years old & $25.00 \%$ & $37.50 \%$ & $37.50 \%$ & & \\
\hline$\geq 85$ years old & $0.00 \%$ & $100.00 \%$ & $0.00 \%$ & & \\
\hline \multicolumn{6}{|l|}{ Gender } \\
\hline Male & $25.44 \%$ & $41.66 \%$ & $33.00 \%$ & .428 & .372 \\
\hline Female & $26.00 \%$ & $44.40 \%$ & $29.60 \%$ & & \\
\hline \multicolumn{6}{|l|}{ Do not visit a family member with dementia during the } \\
\hline No & $28.60 \%$ & $44.90 \%$ & $26.50 \%$ & .917 & .632 \\
\hline Yes & $29.00 \%$ & $35.50 \%$ & $35.50 \%$ & & \\
\hline \multicolumn{6}{|l|}{$\begin{array}{l}\text { Do not allow services of paid caregivers during the COVID-19 } \\
\text { pandemic }\end{array}$} \\
\hline No & $11.10 \%$ & $53.50 \%$ & $35.60 \%$ & 8.981 & .011 \\
\hline Yes & $44.40 \%$ & $27.80 \%$ & $27.80 \%$ & & \\
\hline \multicolumn{6}{|l|}{$\begin{array}{l}\text { Level of burden of the family caregiver during the COVID-19 } \\
\text { pandemic }\end{array}$} \\
\hline Low burden & $27.00 \%$ & $21.60 \%$ & $51.40 \%$ & 17.281 & .002 \\
\hline Medium burden & $8.60 \%$ & $34.30 \%$ & $57.10 \%$ & & \\
\hline High burden & $10.00 \%$ & $37.10 \%$ & $52.90 \%$ & & \\
\hline \multicolumn{6}{|l|}{$\begin{array}{l}\text { Questions about supporting someone with dementia during } \\
\text { the coronavirus outbreak }\end{array}$} \\
\hline $\begin{array}{l}\text { I'm worried that the paid caregivers who come in to help us } \\
\text { might not be able to come. }\end{array}$ & $15.10 \%$ & $39.40 \%$ & $45.50 \%$ & 8.340 & .017 \\
\hline $\begin{array}{l}\text { I need to go outside to pick up supplies for my relative with } \\
\text { dementia but I am worried that I might catch the virus. }\end{array}$ & $56.60 \%$ & $30.40 \%$ & $13.00 \%$ & 10.499 & .005 \\
\hline
\end{tabular}

Notes: This table represents valid percentage of responses on the questionnaire survey specifically designed for this study. The bold represents $p<.05$.

Abbreviations: CDR, Clinical Dementia Rating; AD, Alzheimer's disease.

Characteristics of participating caregivers of persons with $\mathrm{AD}$ or a related dementia are shown in Table 1 , part II. The family was the primary provider of care in $65 \%$, the average age and years of education of family caregivers were 56.21 \pm 14.07 and
$18.46 \pm 6.84$, respectively. Women accounted for $69.23 \%$ of family caregivers. Paid caregivers dedicated higher hours a day to caring for the patients with a CDR score $>2$ compared to CDR score $=1$. 
Sixty percent of family members discontinued all visits to their loved ones, irrespectively of the severity of the dementia, in an attempt to limit the epidemic ( $p$ non-significant). However, only $28.6 \%$ of family caregivers chose to suspend services from paid caregivers. A statistically significant difference was observed in the percentages according to the severity of dementia, with the rate of non-suspension being higher in the more advanced stages $\left(\chi^{2}(2)=8.981\right.$, $p=.011)$.

There was no difference in the level of burden before coronavirus pandemic for family caregivers of persons in the three stages of dementia $(p>.05)$. However, the level of burden of the family caregiver after 4 weeks in quarantine was higher, especially for advanced stages of dementia $\left(\chi^{2}(4)=17.281\right.$, $p=.002$ ).

When we asked family member's main concerns during the epidemic, we found some interesting and significant differences. Relatives of advance dementia subjects were more concern of the possibility of a sick leave of paid caregivers during the pandemic ( $p=.017)$, while relatives of subjects with mild dementia were mainly concerned of the risk of being contagious to the subjects when they assisted them with grocery supplies $(p=.005)$.

\section{Discussion}

This is the initial phase of this research on the effects of mandatory isolation on the care and caregiver stress of subjects with different stages of dementia living in the community.

The sample included a population of older adults with different stages of dementia, approximately $2 / 3$ of the sample had moderate to severe dementia, and as expected, the most common cause of dementia in this older population was $\mathrm{AD}$, followed by mixed AD. Approximately half of the subjects experienced increased anxiety during COVID isolation. Most patients received before the epidemic ambulatory rehabilitation services that included cognitive training, physical therapy, and occupational therapy, interesting subjects with more severe stages of the diseases received more therapies than subjects with milder disease. In most cases, outpatient rehabilitation services were suspended due to confinement independently of the severity of the disease.

As described in other studies done in Latin America, in our sample, caregiving usually relied on family members (Elnasseh et al., 2016). Also, in concordance with published literature, we observed a disparity in gender distribution of family members caring of subjects with $\mathrm{AD}$, with more women taking this role than men. As expected, subjects with a more severe stage of the disease had more hours of paid care due to functional dependence. One of our main findings was the high rate of discontinuation of family visits independently of the severity of the disease, probably related to fear of spreading the disease when visiting subjects. Another finding was that most families of subjects with severe dementia living in the community and not in long-term care facilities decided to continue with the assistance of a paid caregiver. This is likely due to the degree of dependence of this fragile population on caregivers.

Approximately half of the family caregivers reported that subjects with dementia experienced anxiety before the pandemic, irrespectively to the stage of the disease. With the enforced isolation due to COVID 19, this number increased. Loneliness, boredom, and lack of activities are known factors described in the literature to contribute to anxiety (Hwang et al., 2020). Probably all these factors are playing an important role during quarantine. This finding is of concern, and extensive reports on the literature point the negative impact of anxiety on quality of life, functional dependence, and caregiver stress (Savva et al., 2009).

Overall, COVID-19 confinement increased stress caregiver independently of the stage of the dementia, but those caring for severe dementia subjects had more COVID-related stress compared to caregivers assisting subjects with mild dementia. Main concerns of family members varied accordingly to the severity of the dementia. Advanced dementia relatives fear mostly of the absence of the paid caregiver, probably due to subject's absolute dependence on caregivers for survival.

The main limitations of our study included the relatively small size of the sample and the lack of prospectively longitudinal follow-up. Also, there is a limitation related to our statistical analysis that must be considered: $p$ values were not adjusted for multiple comparisons, and type I error rates might be high. We will replicate our study in a larger scale to analyze with more confidence if our results represent a real phenomenon and not just an inflated Type I error. Another limitation is that our main intention was to report in a preliminary way issues related to caregiver's management during the pandemic and no validated instruments to measure variables such as burden of care or anxiety were tested. We will continue to follow this cohort of subjects prospectively to study the health consequences after the end of the isolation period and we will continue our work to test caregivers stress using validated scales.

Social distancing is effective as a global health policy for a disease that so far has no vaccination or approved treatment. In Argentina, main efforts of the authorities had been to identity, isolate, and treat 
sick individuals and social services and help was mainly assigned to the care of symptomatic and severely ill patients and families. In older adults with dementia, we observed a rise in clinically relevant complications due to social isolation and we fear that this population may suffer more functionally dependence, dementia progression, and worsening of their quality of life. Our results showed that caregivers are already grappling with rising stress levels caused by the pandemic. We urge social services and governments to address specific interventions for the population of subjects with dementia and their relatives. A partnership between departments of public health, care workers, and families must be planned to guarantee continuity of care. A post-COVID-19 reality must be considered to ameliorate the real impact of mandatory social isolation in vulnerable populations with cognitive decline.

\section{Conflict of interest}

None.

\section{Description of authors' roles}

The study was designed and conceived by Gabriela Cohen (GC), María Julieta Russo (MJR), Jorge Campos (JC), and Ricardo F. Allegri (RFA). Recruitment of participants and data collection were undertaken by GC and MJR. Statistical analysis was overseen by RFA, and analysis was undertaken by GC and MJR. The manuscript was prepared by GC and critically reviewed and approved by all authors.

\section{Acknowledgments}

The authors would like to thank the caregivers and relatives who took time to participate in this survey.

\section{References}

Austrom, M. G., Boustani, M. and Lamantia, M. A. (2018). Ongoing medical management to maximize health and well-being for persons living with dementia.

Gerontologist, 58(2014), S48-S57. https://doi.org/10.1093/ geront/gnx147

Brooks, S. K. et al. (2020). The psychological impact of quarantine and how to reduce it: rapid review of the evidence. Lancet, 395(10227), 912-920. https://doi.org/10 $.1016 /$ S0140-6736(20)30460-8

Elnasseh, A. G. et al. (2016). Family dynamics and personal strengths among dementia caregivers in Argentina. International fournal of Alzheimer's Disease, 1, 1-10. https:// doi.org/10.1155/2016/2386728

Emami, A., Javanmardi, F., Pirbonyeh, N. A. and Akbari, A. (2020). Prevalence of underlying diseases in hospitalized patients with COVID-19: a systematic review and meta-analysis. Archives of Academic Emergency Medicine, $8(1)$, e 35.

Hughes, C., Berg, L., Danziger, W., Coben, L. and Martin, R. (1982). A new clinical scale for the staging of dementia. British fournal of Psychiatry, 140, 566-572.

Hughes, T. B., Black, B. S., Albert, M. and Glitin, L. (2014). Correlates of objective and subjective measures of caregiver burden among dementia caregivers: influence of unmet patient and caregiver dementia-related care needs. International Psychogeriatrics, 26(11), 1875-1883. https:// doi.org/10.1017/S1041610214001240

Hwang, T.-J., Rabheru, K., Peisah, C., Reichman, W. and Ikeda, M. (2020). Loneliness and social isolation during the COVID-19 pandemic. International Psychogeriatrics, 1-4. Advance online publication. https://doi .org/10.1017/S1041610220000988

Ministerio de la Nación (2020). República Argentina. Decreto de Necesidad y urgencia 260/2020.

Nimon, K. (2014). Explaining differences between retrospective and traditional pretest self-assessments: competing theories and empirical evidence. International Fournal of Research E Method in Education, 37(3), 256-269. https://doi.org/10.1080/1743727X.2013.820644

Savva, G. M. et al. (2009). Prevalence, correlates and course of behavioral and psychological symptoms of dementia in the population. British fournal of Psychiatry, 194(3), 212-219. https://doi.org/10.1192/bjp .bp.108.049619 